\title{
Alcance del estereotipo femenino presentado en la telenovela "La Rosa de Guadalupe" en jóvenes estudiantes de telesecundaria.
}

Tejeda Noriega Montserrat ${ }^{1}$

Gutiérrez Jiménez Lizbeth ${ }^{2}$

Jiménez González María Fernanda ${ }^{3}$

Rodríguez Palacios Eduardo ${ }^{4}$

\section{Resumen}

Esta investigación muestra el impacto que tuvo el programa "La Rosa de Guadalupe" en los adolescentes entre 13 y 15 años de edad en una pequeña comunidad en San Agustín Tlaxiaca, área periférica a Pachuca, Hidalgo con el objetivo de analizar los efectos negativos que este programa les puede producir y hacerles saber que no toda la información proporcionada es necesariamente cierta. Investigación concluida en 2013.

Para esta investigación, utilizamos el método cualitativo, las conclusiones obtenidas muestran cómo la población estudiada está imitando lo que ve en el programa, tales como las actitudes, comportamientos y vestimenta que tienen los actores, lo cual impacta de manera central en una identificación principalmente con los personajes de las víctimas.

\section{Introducción}

\section{Problemática}

Tipo de manipulación que ejerce el estereotipo femenino de la telenovela La rosa de Guadalupe en los estudiantes del $2^{\circ}$ grado de la telesecundaria de Tilcuautla durante el semestre enero - junio 2013.
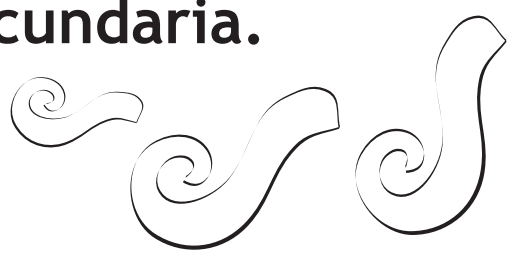

\section{Objetivos}

\section{General:}

Identificar el prototipo ideal de lo femenino que ejerce el programa "la Rosa de Guadalupe" entre los estudiantes de la telesecundaria.

\section{Específicos}

1. Saber las situaciones particulares con las que se identifican los alumnos de $2^{\mathrm{O}}$ de la telesecundaria de Tilcuautla con el programa de La rosa de Guadalupe.

2. Clasificar el contenido del programa de La rosa de Guadalupe.

3. Identificar los estereotipos que más fuerza tienen sobre los alumnos de $2^{\mathrm{o}}$ de la telesecundaria de Tilcuautla.

\section{Preguntas}

1. ¿Por qué los jóvenes de $2^{\mathrm{o}}$ año de la telesecundaria de Tilcuautla eligen el programa de La Rosa de Guadalupe?

2. ¿Qué efecto físico causa en el estereotipo de vestir y afecta en las niñas el programa La rosa de Guadalupe sobre los estudiantes del $2^{\mathrm{O}}$ año de la telesecundaria de Tilcuautla?

3. ¿Qué efecto conductual y psicológico causa el programa La rosa de Guadalupe en las niñas estudiantes del $2^{\circ}$ año de la telesecundaria de Tilcuautla?

1 glow_tejeda@hotmail.com. Sexto semestre de la Licenciatura en Ciencias de la Comunicación, ULSA Pachuca.

2 liz_gtz_j@hotmail.com. Sexto semestre de la Licenciatura en Ciencias de la Comunicación, ULSA Pachuca.

3 fda.jimenez@gmail.com. Sexto semestre de la Licenciatura en Ciencias de la Comunicación, ULSA Pachuca.

4 edward.rp94@gmail.com. Sexto semestre de la Licenciatura en Ciencias de la Comunicación, ULSA Pachuca. 


\section{Justificación}

Una urgencia que se presenta en nuestra problemática es la de una "educación de calidad", ya que es muy importante que los programas que vean los jóvenes tengan un contenido de calidad.

Hoy en día muchos de los programas televisivos tienen un contenido que no es nada favorable para los jóvenes. Nosotros nos enfocaremos a analizar el contenido del programa La rosa de Guadalupe para observar cuáles son los factores negativos y los positivos y ver el contenido del programa para analizar si es de calidad o no.

Con este programa se beneficiarán los estudiantes de la telesecundaria de Tilcuautla.

\section{Marco teórico}

\section{Marco histórico}

En 1933 la "teoría hipodérmica" atribuye a los medios una habilidad de manipular las ideas de las personas. Los medios se consideraban capaces de moldear directamente la opinión pública y lograr que las masas adoptaran casi cualquier punto de vista. Gesbell; ésta fue una de las primeras teorías que sacaron a la luz, la capacidad de manipulación que tiene la televisión (M. Wolf en La investigación de la comunicación de masas. Crítica y perspectivas, Paidós, Barcelona 1991).

En 1940, sobre como las personas se encuentran con los medios y cómo reaccionan ante ellos. Berelson.

En 1948, " ¿Quién?dice qué?a través de qué canal a quién y con qué efecto?"

Aristóteles fue el primero que dio a conocer la triada de la comunicación, fue hasta la Segunda Guerra Mundial, que Lasswell notó que tenía que haber un efecto en lo que se dice y que siempre es a través de un canal (H. Lass-

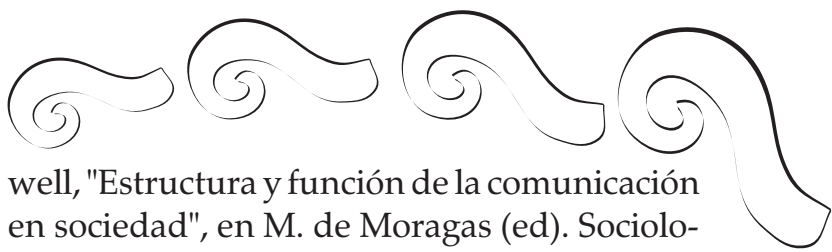

gía de la comunicación de masas, Gustavo Gili, Barcelona 1986, Vol. II)

En 1951 "Entre la conciencia y la existencia están las comunicaciones, que influyen en esa conciencia que los hombres tienen de su existencia" Wright Mills, Esta opinión nos deja entender que mucho de lo que decimos y pensamos tiene su origen en la televisión $\mathrm{u}$ otros medios de comunicación.

En 1965 "lo que no existe en los medios, no sucedió nunca" Walter Lippman, lo que no sale en los medios de comunicación es desconocido por la mayoría. Esta frase de Lippman tiene mucha verdad.

En 1975 los medios de comunicación buscan satisfacer sus necesidades a través de nuestra gratificación, aunque estos después nos generen problemas. Blumer y Katz, (De Flour, Melvin. Teorías de la comunicación de masas. Ed Paidos, 1997).

En 1979 "los medios se centran más en la estructura económica que en el contenido ideológico de los medios. Las consecuencias de esto pueden observarse en la reducción de las fuentes independientes, la concentración en los mayores mercados, desprecio de sectores más pobres en audiencia. "Garnham Murdoch., la manipulación que se ve en la televisión es debido a las necesidades económicas. (Mc Quail, Denis. Introducción a la teoría de la comunicación de masas. Ed. Paidos. Pag 93-94)

En 1990 "el proceso de influencia necesita tiempo"Katz

En 1995 "los medios suministran a la gente las palabras y las frases que pueden utilizar para defender un punto de vista. Si la gente no encuentra expresiones habituales, repetidas con frecuencia, a favor de su punto de vista, cae en el silencio, se vuelve muda" Noelle Neumann. Normalmente nosotros hablamos de lo 


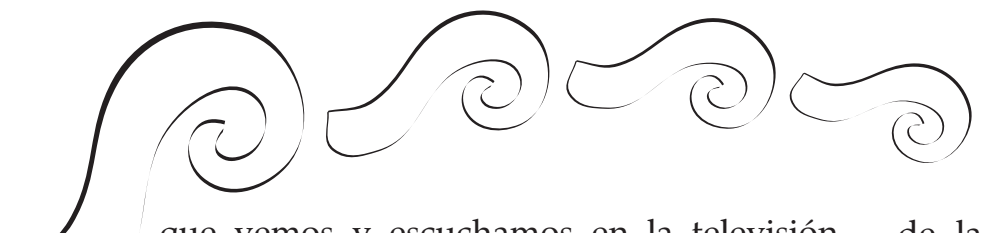

que vemos y escuchamos en la televisión, nuestra opinión parte de ahí para saber qué es lo que está bien o mal, o qué está a la moda o no. (Portillo Sánchez Maricela, Espacios de comunicación. Universidad).

En 1996 "el fantasma de la opinión pública es el resultado complejo de procesos dinámicos no lineales que ocurren en la mente de los individuos como resultado de la interacción social y la comunicación." Howard Lavine y Bibb Latane (Portillo Sánchez Maricela, Espacios de comunicación. Universidad Iberoamericana, México 1998).

\section{Marco conceptual}

Manipulación. "La palabra manipular implica o refiere a la capacidad que tienen algunas personas para manejar determinadas cosas, trabajos, objetos, especialmente aquellos delicados o que requieren de precisión para su funcionamiento."(Gloria Robles, h t t p://www.proyectopv.org/1 verdad/manipulacion.htm)

Manipular es el poder o capacidad que tienen algunas personas para llevar a cabo algunas actividades y de forma particular a los que necesitan más atención para su funcionamiento.

Televisión. "El arte de producir instantáneamente a distancia una imagen transitoria, visible de una escena real o filmada por medio de un sistema electrónico de telecomunicación." (Jorge González Treviño, http://definicion.de/television/)

La televisión es cuando se producen en el momento o de lejos diferentes imágenes, dichas imágenes pueden ser reales o grabadas mediante sistemas electrónicos.

Programa La Rosa de Guadalupe es un programa mexicano alusivo a la religión católica romana y en específico a la Virgen de Guadalupe. Se transmite actualmente por el Canal de las Estrellas. (Televisa, http://televisa.esmas.com/la-rosa-de-guadalupe/)

La rosa de Guadalupe es un programa mexicano de inspiración católica. Hacen alusión a los milagros que hace la Virgen de Guadalupe.

Prototipo. "Ejemplar más perfecto y modelo de una virtud, vicio o cualidad." (RAE, http://lema.rae.es/drae/?val=prototipo)

Un prototipo es una imagen perfecta que se crea de algo o alguien. Se crea el prototipo y se cree que es lo más perfecto.

Feminidad es el conjunto de cualidades que se manifiestan en mayor medida en las mujeres en una cultura particular. Es un concepto que alude a los valores, características y comportamientos tanto aprendidos, como a características específicamente biológicas. (RAE. http://lema.rae.es/drae/?val=feminidad)

La feminidad son las características que tiene en general una mujer, esto puede ser aprendido y también se tienen de manera biológica. Estos pueden variar dependiendo la cultura.

\section{Marco teórico}

Todos los estudios o teorías del siglo XX como: La teoría de la Sociedad de masas de Nietszche o La teoría de la espiral del silencio de Tocqueville sobre los medios de comunicación eran acerca de los efectos de los mismos sobre las masas. Para fines de nuestra investigación nos enfocaremos a las teorías que surgen en la década de los 60 sobre lo que hacen los individuos, ya no las masas con los medios de comunicación buscando mensajes para sus necesidades.

Para esta investigación nos basaremos en la teoría formulada por Elihu Katz, sociólogo inglés, "La teoría de los usos y gratificaciones", Estados Unidos 1960 que dice:

"Cada individuo selecciona los estímulos a los que quieren responder, atendiendo a causas como sus valores, intereses y funciones sociales. Por tanto, más que ser los 
medios los que dicen al espectador qué ver, son los usuarios de forma activa los que lo deciden, atendiendo a sus necesidades y la gratificación que les proporcionen." (Katz, Elihu, en Defleur, Melvin, Teorías de la comunicación de masas, México, Ed Pároleo 1997. Pp: 247-250)

"Cuestiona la relación directa entre estímulo y respuesta, por lo que atiende al hecho de que cada uno de los destinatarios de un mismo mensaje, viene precedido por un contexto el cuál condiciona el efecto de dicho mensaje. Por tanto, no son solo los estímulos los que ponen en marcha el proceso comunicativo, sino los propios receptores al elegir el contenido e interpretarlo. Los estímulos generan unos efectos tan solo si el individuo quiere responder a ellos." (Katz, Elihu, en A y M, Maklelart, Historia de las teorías de la comunicación, Ed Paidós. Española 1997 pp102-105)

Los medios compiten entre ellos, y al mismo tiempo con otras fuentes, para lograr la atención del público que busca satisfacer sus necesidades" (Katz, Elihu, en A y M, Maklelart, Historia de las teorías de la comunicación, Ed Paidós. Española 1997 pp102-105)

Con base a estos postulados podremos descubrir la causa principal por la cual a los niños de segundo grado de la telesecundaria de Tilcuautla les gusta ver la telenovela de $\mathrm{La}$ rosa de Guadalupe y qué es lo que buscan en ella.

Para Katz era necesario satisfacer cuatro necesidades:

1. Necesidades inter-psicológicas: que se refiere al integrarse y a la sustitución de la compañía, así como reforzar los estándares sociales de pertenencia.

2. Necesidades intra-psicológicas: que se refiere al identificarse, al autoconocimiento y a la identificación de valores que se presentan, así como relacionarse con algún estereotipo.

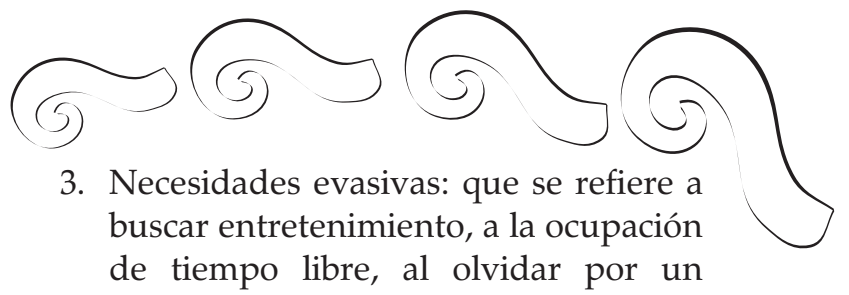

momento los problemas.

4. Necesidades cognitivas: que se refiere a informarse, al autoaprendizaje, y al mantenerse al tanto de lo que ocurre en el entorno. (Defleur, Melvin, Teorías de la comunicación de masas, México, Ed Pároleo 1997. Pp: 247-250. http://es.wikipedia.org/wiki/Teor\%C3

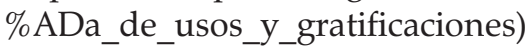

Para nuestra investigación será muy útil seguir estos postulados y clasificaciones ya que podremos analizar las razones y causas por las cuales este programa es tan popular entre los estudiantes de la escuela antes mencionada y que tanto impacto tiene en la vida de los mismos.

\section{Marco referencial}

Dentro del marco referencial se encontraron muy pocos resultados debido a que la serie a estudiar es relativamente nueva y al ser lanzada en 2008 por la cadena de medios de comunicación más importante de habla hispana (Televisa) tiende a ser sumamente controlada en cuanto a la información y críticas que se pudieran realizar en torno a este programa de melodrama, telerrealidad y docudrama.

Actualmente no existe ninguna investigación realizada a profundidad acerca de la manipulación que ejerce "La rosa de Guadalupe" en las personas. Existen múltiples investigaciones sobre programas similares producidos por esta televisora y demasiadas críticas acerca de la misma a partir de 1968, pero no se encontró evidencia de alguna investigación parecida a nuestro tema en periódicos, internet o algún documento escrito.

\section{Método.}

\section{Enfoque cualitativo}

Utilizaremos la metodología cualitativa porque recaudaremos datos y analizaremos el 


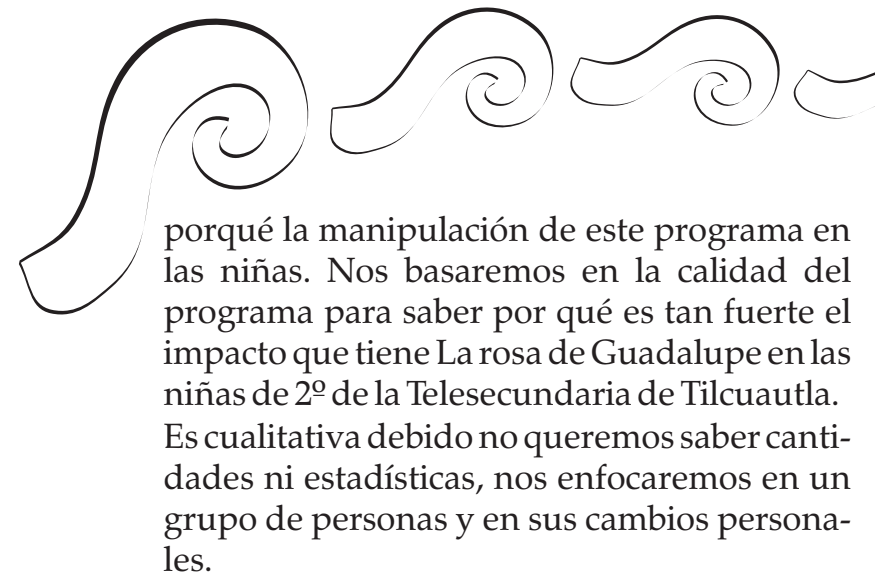

\section{Alcance descriptivo}

Utilizaremos la descriptiva porque estudia sobre todo lo social. Nos enfocaremos en una problemática concreta para llegar al porqué de los comportamientos de las niñas al ver el programa La rosa de Guadalupe.

\section{Diseño no experimental}

Nuestro diseño es no experimental porque se basará en hechos que ya ocurrieron, no se modificarán las variables. Con los hechos pasados actualizaremos los datos y llevaremos a cabo la investigación.

\section{Contexto}

Realizamos nuestra investigación con los estudiantes del 2a de la telesecundaria no 196 de Tilcuautla ubicada en el estado de Hidalgo. Sujeto, universo, muestra:

Universo: la población de la telesecundaria de Tilcuautla es de 65 estudiantes.

Sujeto: son estudiantes del $2^{\circ}$ grado de la telesecundaria de Tilcuautla. e encuentran en edades entre los 13 y 14 años. La mayoría de estos jóvenes son de escasos recursos.

Muestra: estudiantes del $2^{\circ}$ grado de la telesecundaria de Tilcuautla que cuenta con 40 alumnos.

Algunas de sus características generales son:

- Prueban los límites, actitud de "lo sé todo"

- Se identifican con un adulto que admi- ran; pueden reflejar ejemplos de ese adulto.

- Vulnerables, emocionalmente inseguros, temen el rechazo, cambios en el estado de ánimo.

- Los cuerpos están comenzando a pasar por cambios físicos que afectan la apariencia personal.

Algunas de las características físicas son:

- Un poco de coordinación muscular es buena.

- El crecimiento óseo todavía no está completo.

- Están muy preocupados con respecto a su apariencia y muy vergonzosos acerca del crecimiento.

- Los hábitos alimenticios o de sueño pueden ser malos o inconsistentes, lo cual puede resultar en bajos niveles de energía.

- Las jovencitas pueden comenzar la menstruación.

\section{Supuesto}

Suponemos que las niñas de segundo grado de secundaria de la escuela de Tilcuautla, imitan las actitudes, maneras de vestir y maneras de hablar, al idealizar estos papeles como un ejemplo a seguir, para obtener los mismos resultados que muestra la telenovela de $\mathrm{La}$ rosa de Guadalupe.

\section{Procedimiento}

1. Ir a la telesecundaria a pedir autorización para aplicar los instrumentos de la investigación a los jóvenes.

2. Un vez que se cuente con la autorización, continuamos con la aplicación del instrumento.

3. Finalmente juntamos los resultados que nos arrojaron las respuestas de los jóvenes de acuerdo a las encuestas. 
Formato de instrumento de recolección

\begin{tabular}{r|c|c|}
\cline { 2 - 3 } Sexo: & Hombre. & 20 \\
\cline { 2 - 3 } & Mujer. & 16 \\
\cline { 2 - 3 } Ocupación: & Estudiantes & 36 \\
\cline { 2 - 3 } Edad: & 13 años & 17 \\
\cline { 2 - 3 } & 14 años & 15 \\
\cline { 2 - 3 } & 15 años & 4 \\
\hline
\end{tabular}

¿Has visto el programa la Rosa de Guadalupe?

\begin{tabular}{|r|r|}
\hline Siempre & 3 \\
\hline A veces & 32 \\
\hline Nunca & 1 \\
\hline
\end{tabular}

Con base en las historias que se muestran en este programa ¿Cuál es el personaje con quién te identificas siempre?

\begin{tabular}{|r|c|}
\hline La víctima & 12 \\
\hline Los familiares & 19 \\
\hline El héroe & 3 \\
\hline El villano. & 2 \\
\hline
\end{tabular}

¿Por qué decides ver este programa y no cualquier otro de los que transmiten en la televisión?

\begin{tabular}{|r|c|}
\hline Es el único canal que tengo & 0 \\
\hline Me gusta el programa & 11 \\
\hline Me enseña valores & 7 \\
\hline Por distracción & 18 \\
\hline
\end{tabular}

¿Crees que el estereotipo que muestra este programa acerca de la mujer mexicana es...?

\begin{tabular}{|r|c|}
\hline Culta y educada & 25 \\
\hline Inculta y mal educada & 1 \\
\hline Amorosa pobre & 7 \\
\hline Rica sin valores & 3 \\
\hline
\end{tabular}

¿Cuáles son los principales temas que aborda el programa?

\begin{tabular}{|r|r|}
\hline Discriminación & 8 \\
\hline Violencia & 23 \\
\hline Bullying & 5 \\
\hline
\end{tabular}

¿Cuál es el tipo de personaje del cual has copiado algo de su forma de ser, vestir, arreglarse o que simplemente te ha llamado la atención?

\begin{tabular}{|r|r|}
\hline Personaje rico & 5 \\
\hline El que hace daño & 3 \\
\hline Te muestras indiferente & 15 \\
\hline La víctima & 13 \\
\hline
\end{tabular}

¿Cuál crees que sea la razón por la que tú u otros compañeros tuyos han cambiado su arreglo físico al copiar la moda de este programa?

\begin{tabular}{|r|r|}
\hline Para no sentirme menos o fuera de lugar & 5 \\
\hline Para estar a la moda & 3 \\
\hline Porque quisiera identificarme con ese estilo & 15 \\
\hline
\end{tabular}

¿Al terminar de ver un capítulo de este programa qué es lo primero que piensas?
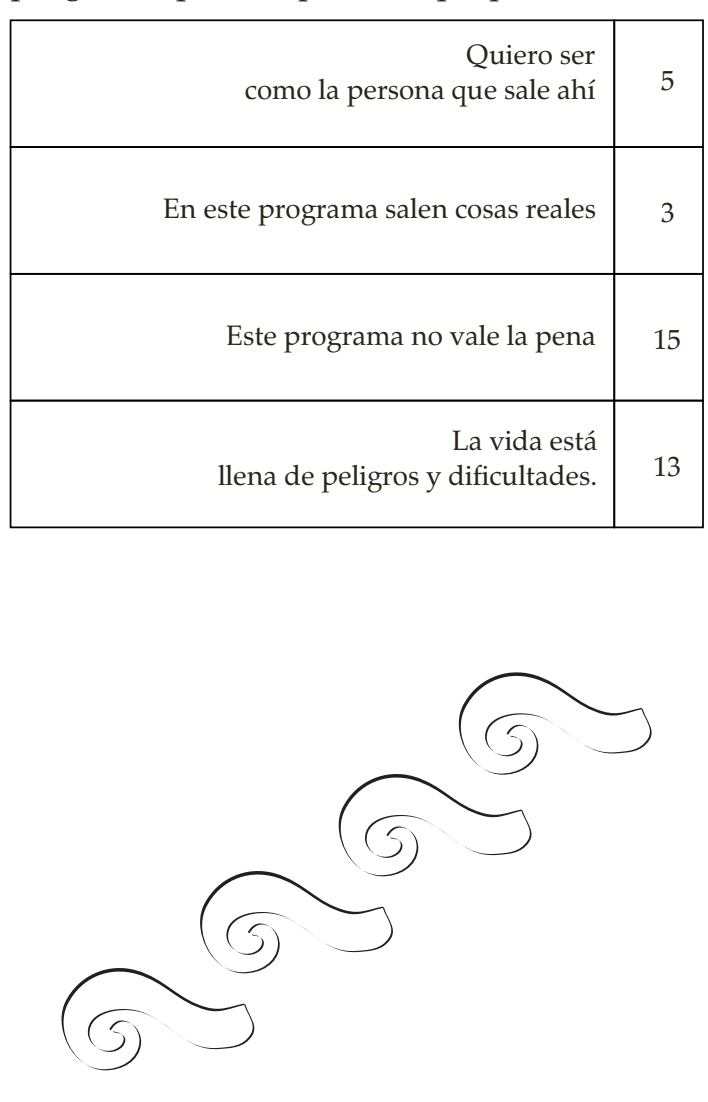
Sexo

En la encuesta realizada el día 29 de mayo del 2013 en el ámbito de género arrojaron que hay más hombres que mujeres estudiando el segundo año de telesecundaria en la comunidad de Tilcuautla.

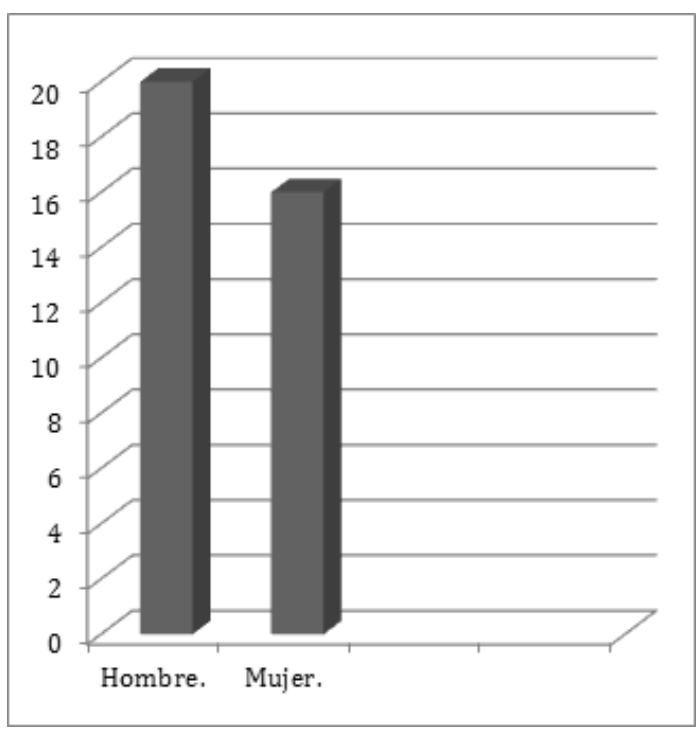

Esto se debe a que en la población mexicana existe un rango muy leve en cuando a mayor número de hombres o mujeres en una población. En este caso un 56\% fueron hombres y un $44 \%$ mujeres.

Nosotros pensamos que hoy en día, la mayor parte de la población es del sexo masculino.

Según el INEGI la población de Hidalgo es mayoritaria en mujeres.

http://www.inegi. org.mx/movil/

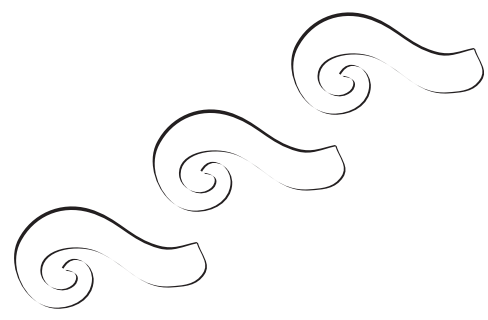

Edad

En la encuesta realizada el día 29 de mayo del 2013 en el ámbito de edad arrojaron que el rango promedio está entre los 13 y 14 años.

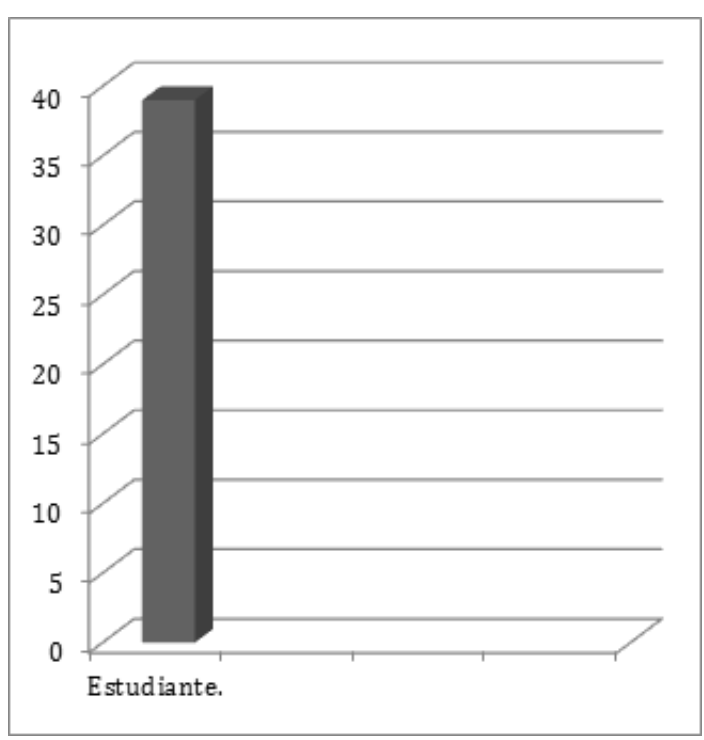

Esto se debe a que en esta comunidad existe un alto grado de pobreza y por lo tanto los alumnos no tienen la oportunidad de inscribirse a tiempo en las escuelas y ante a la mala calidad de la educación tienden a reprobar consecutivamente $\mathrm{y}$ es por esta razón que existen alumnos con dos años de diferencia.

Nosotros creemos que de igual forma, no hay interés de los jóvenes y de sus padres porque tener una educación satisfactoria.

Según el INEGI, los jóvenes entre 12 y 15 años se dedican a estudiar el segundo grado de secundaria o telesecundaria.

http://www.inegi.org.mx/movil/

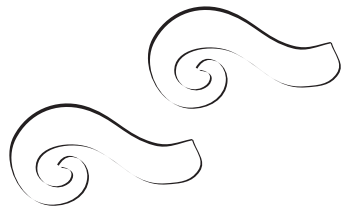




\section{Ocupación}

En la encuesta realizada el día 29 de mayo del 2013 en el ámbito de ocupación arrojaron que de 36 alumnos, el $100 \%$ se dedican a ser estudiantes.

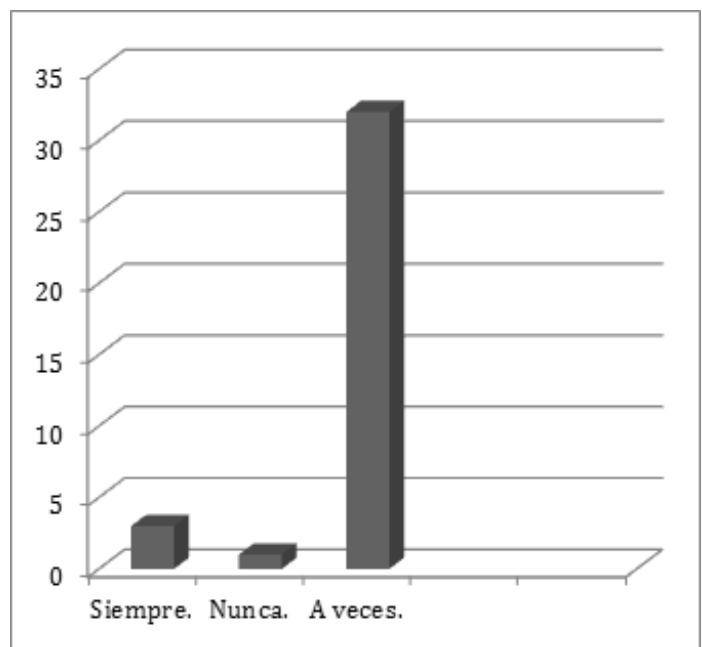

Esto se debe a que en esta comunidad no existen oportunidades de trabajo para alumnos de su edad y por lo tanto lo único pueden hacer es estudiar o ayudar a sus padres.

Debido al exceso de jóvenes, en el lugar no hay muchas oportunidades de trabajo.

Según el INNEGI los jóvenes entre 12 y 15 años se dedican a estudiar el segundo grado de secundaria o telesecundaria.

http://www.inegi.org.mx/movil/

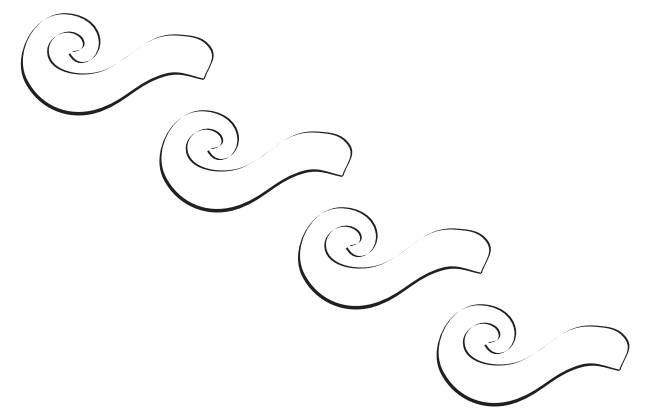

\section{Conocimiento del programa}

En la encuesta realizada el día 29 de mayo del 2013 en el ámbito de conocimiento del programa arrojaron que la gran mayoría de los estudiantes ven el programa de manera cotidiana pero no siempre.

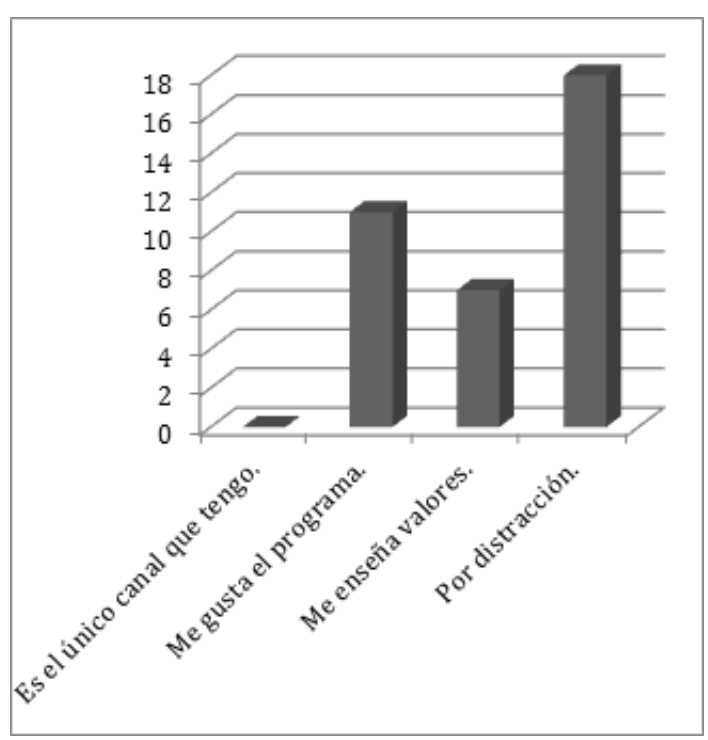

Esto se debe a que en esta comunidad los estudiantes se dedican únicamente a ser estudiantes, pero deben ayudar en las labores del hogar y/o en el trabajo familiar.

De igual forma es porque las madres de familia tienden mucho a ver este tipo de programación.

Según la programación basada en los títulos de Televisa.

http://www.televisa.com

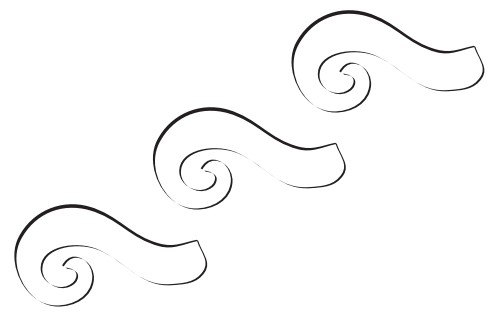




\section{¿Por qué deciden ver este programa?}

En la encuesta realizada el día 29 de mayo del 2013 en el ámbito del porqué deciden ver este programa, arrojaron como resultado que la mayoría ve este programa por distracción y porque les parece agradable pero nadie porque sea el único canal que puedan sintonizar.

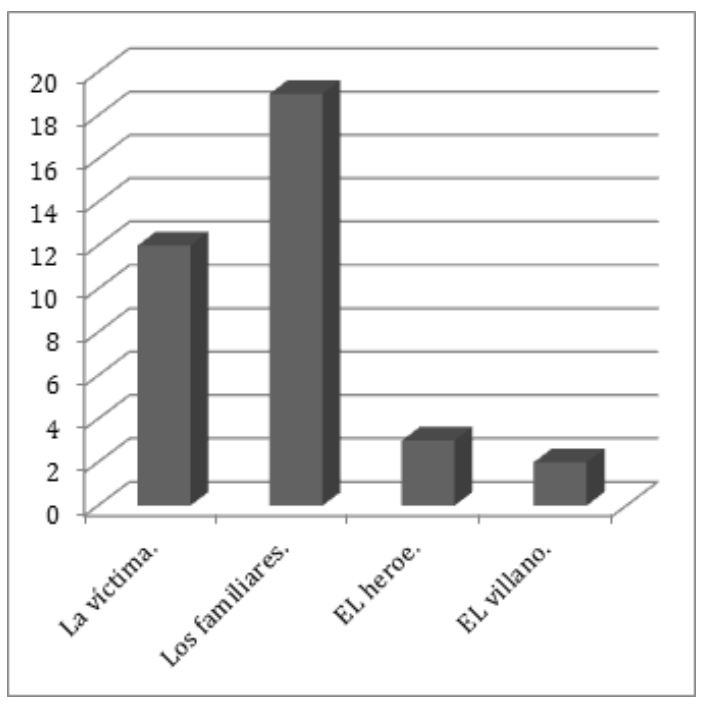

Esto se debe a que en esta comunidad, sí se tiene una situación de pobreza pero los medios para colocar televisión satelital han llegado al alcance de los habitantes y los estudiantes lo ven porque se transmite en un horario de relajación después de la escuela y antes de cenar.

Ellos ocupan su tiempo libre para ver esta programación.

Según Elihu Katz, sociólogo inglés, "La teoría de los usos y gratificaciones", Estados Unidos 1960.

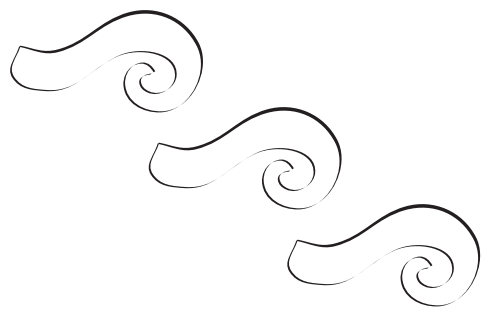

\section{Personaje de identificación}

En las encuestas realizadas el día 29 de mayo del 2013 en el ámbito del personaje de identificación arrojaron que los jóvenes se identifican principalmente con los familiares y la víctima en los capítulos de La rosa de Guadalupe.

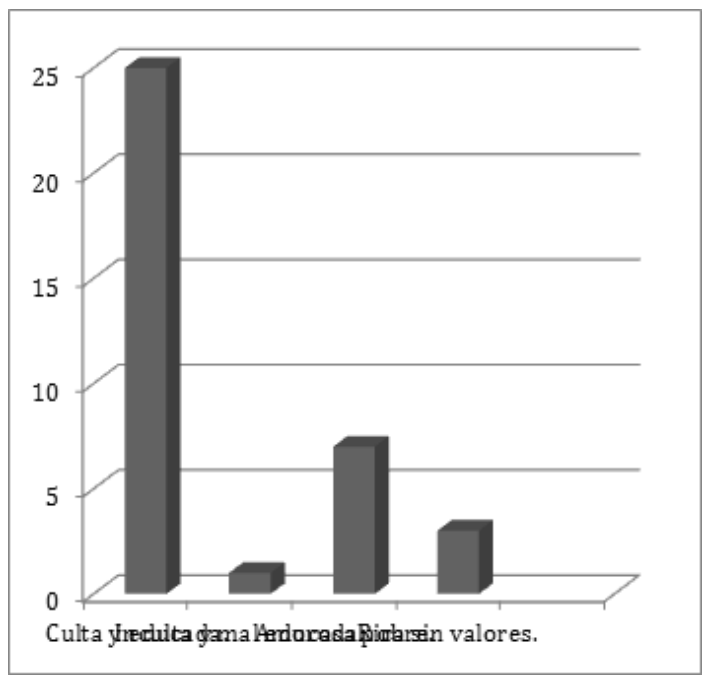

"Cada individuo selecciona los estímulos a los que quieren responder, atendiendo a causas como sus valores, intereses y funciones sociales. Por tanto, más que ser los medios los que dicen al espectador qué ver, son los usuarios de forma activa los que lo deciden, atendiendo a sus necesidades y la gratificación queles proporcionen." (Katz, Elihu)

Defleur, Melvin, Teorías de la comunicación de masas, México, Ed Pároleo 1997. Pp: 247-250.

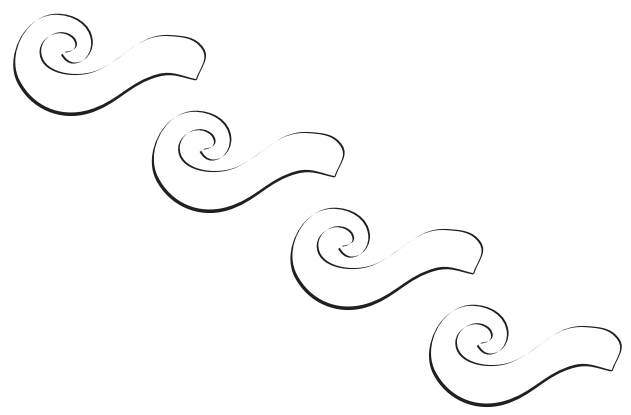


Estereotipo de la mujer mexicana que se muestra en este programa

En la encuesta realizada el día 29 de mayo del 2013 en el ámbito de cómo ven los alumnos el estereotipo de la mujer mexicana, arrojaron que la gran mayoría la ven como una mujer culta y educada.

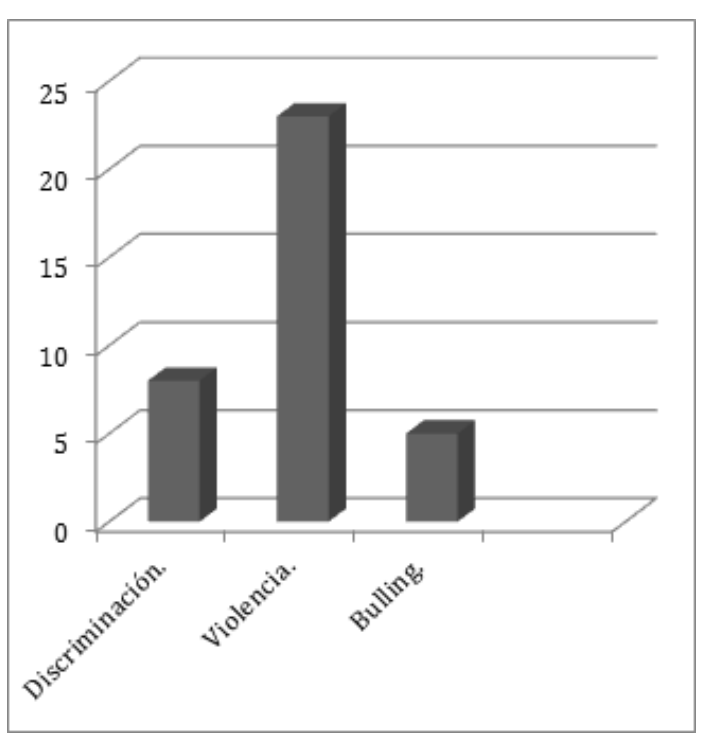

Esto se debe a que los estudiantes se enfoncan principalmente en las mujeres que les llaman la atención o aspiran a ser como ellas, que en este caso serían aquellas que tienen los personajes de mujeres ricas con valores, lo cual es muy diferente a una mujer culta y educada.

Según el INNEGI la mayoría de las mujeres mexicanas se dedican al hogar.

http://www.inegi.org.mx/movil/

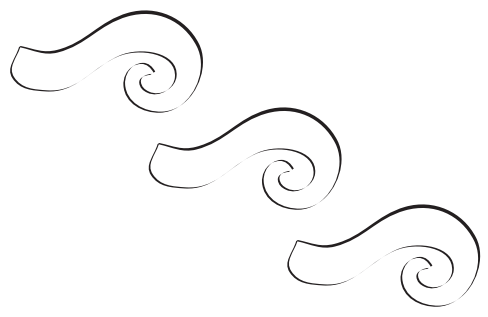

\section{Principales temas abordados en el programa}

En la encuesta realizada el día 29 de mayo del 2013 en el ámbito de principales temas abordados en el programa, arrojaron que los temas que tienen más peso en el programa son sobre violencia.

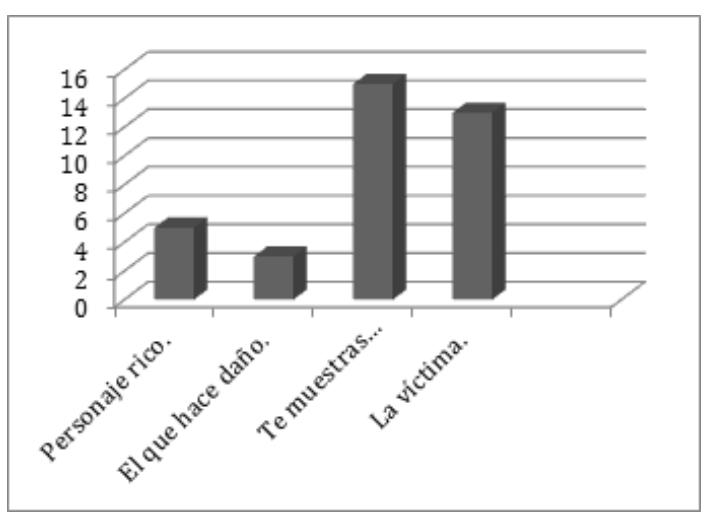

Esto se debe a que es el tema se plante durante el programa es el que está causando más ruido en la sociedad y por lo consiguiente produce más audiencia.

“Cuestiona la relación directa entre estímulo y respuesta, atendiendo al hecho de que cada uno de los destinatarios de un mismo mensaje, viene precedido por un contexto el cuál condiciona el efecto de dicho mensaje. Por tanto, no son solo los estímulos los que ponen en marcha el proceso comunicativo, sino los propios receptores al elegir el contenido e interpretarlo. Los estímulos generan unos efectos tan sólo si el individuo quiere responder a ellos." (Katz, Elihu)

A y M, Maklelart, Historia de las teorías de la comunicación, Ed Paidós. Española 1997 pp102-105

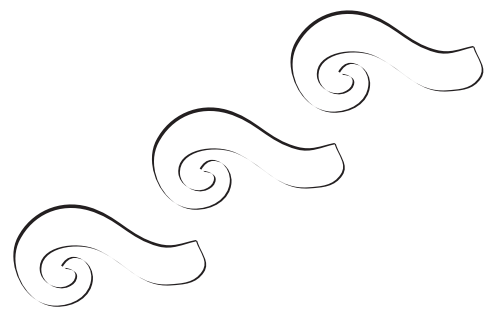




\section{Tú personaje modelo}

En la encuesta realizada el día 29 de mayo del 2013 en el ámbito sobre el personaje modelo, arrojaron que existe poca diferencia entre los que se muestran indiferentes y los que copian cosas de la víctima.

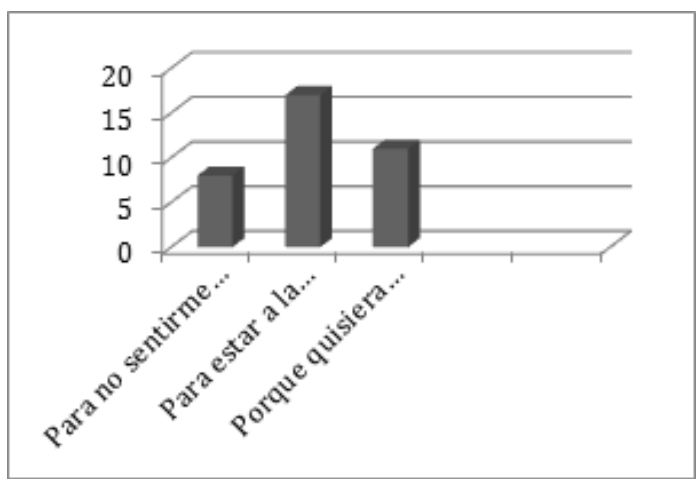

Esto se debe a que los estudiantes tratan de evadir la realidad al verse reflejados como las víctimas y se muestran indiferentes, aunque también existe un porcentaje parecido que dice abiertamente que ha copiado cosas de la víctima debido a que es con el modelo con el que se identifican.

"Cada individuo selecciona los estímulos a los que quieren responder, atendiendo a causas como sus valores, intereses y funciones sociales. Por tanto, más que ser los medios los que dicen al espectador qué ver, son los usuarios de forma activa los que lo deciden, atendiendo a sus necesidades y la gratificación que les proporcionen." (Katz, Elihu)

Defleur, Melvin, Teorías de la comunicación de masas, México, Ed Pároleo 1997. Pp: 247250 .

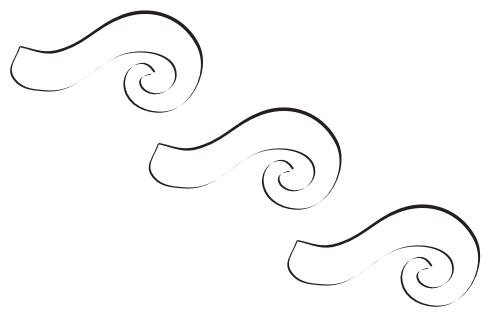

\section{Razón de cambio en arreglo físico}

En la encuesta realizada el día 29 de mayo del 2013 en el ámbito de la razón de cambio en el arreglo físico, arrojaron que la gran mayoría solo lo hace por estar a la moda.

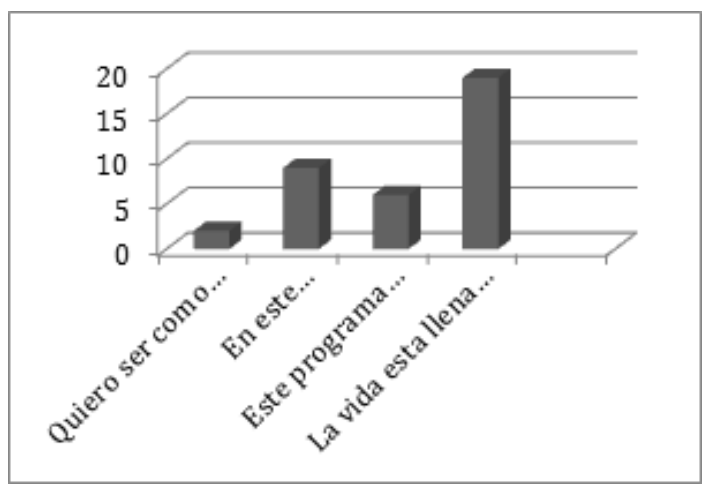

Esto se debe a que la televisión impone lo que ellos y todos nosotros le llamamos moda, que no es otra cosa más que la forma peculiar y diferente de vestir de una persona que alguien que es líder de opinión o que tiene influencia en los demás dice que está bien y entonces esto se acepta y se produce la moda.

"Cada individuo selecciona los estímulos a los que quieren responder, atendiendo a causas como sus valores, intereses y funciones sociales. Por tanto, más que ser los medios los que dicen al espectador qué ver, son los usuarios de forma activa los que lo deciden, atendiendo a sus necesidades y la gratificación que les proporcionen." (Katz, Elihu)

Defleur, Melvin, Teorías de la comunicación de masas, México, Ed Pároleo 1997. Pp:247-250.

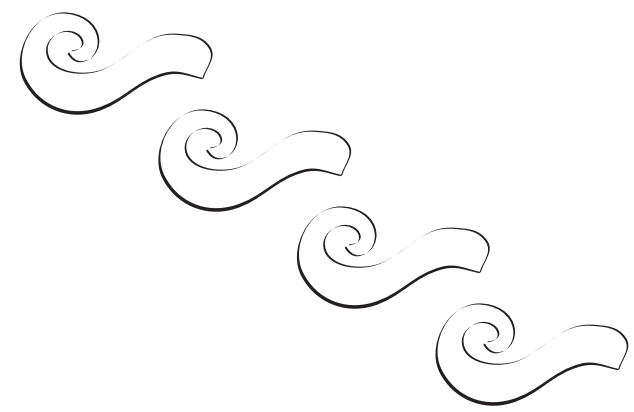




\section{Mensaje final}

En la encuesta realizada el día 29 de mayo del 2013 en el ámbito de mensaje final, arrojaron que este programa le deja a la gran mayoría un mensaje negativo el cual explícitamente es "la vida está llena de peligros y dificultades".

Esto se debe a que los temas abordados se enfocan mucho en la violencia.

\section{Conclusiones}

"Cuestiona la relación directa entre estímulo y respuesta, atendiendo al hecho de que cada uno de los destinatarios de un mismo mensaje, viene precedido por un contexto el cuál condiciona el efecto de dicho mensaje. Por tanto, no son solo los estímulos los que ponen en marcha el proceso comunicativo, sino los propios receptores al elegir el contenido e interpretarlo. Los estímulos generan unos efectos tan sólo si el individuo quiere responder a ellos." (Katz, Elihu)

A y M, Maklelart, Historia de las teorías de la comunicación, Ed Paidós. Española 1997 pp102-105

Identificar cuál es la razón principal por la que los alumnos de segundo grado de la telesecundaria 196 de Tilcuautla ven la telenovela de La rosa de Guadalupe, obtuvimos dos resultados: el primero es que ven la telenovela por distracción y la segunda porque les gusta realmente el programa. Con estos resultados comprobamos la teoría de Elihu Katz, "La Teoría de los usos y gratificaciones", (Estados Unidos 1960). Que nos menciona que las masas eligen el contenido y la información que mayor les convenga y que logre producir en ellos alguna satisfacción. En una plática con los niños nos mencionaron que el programa les ayuda a saber cómo actuar ante diferentes problemáticas que se presentan en su vida diaria. Lo que nos lleva a la siguiente pregunta, la cual resulta que fue sobre el efecto que causan los estereotipos, pues con

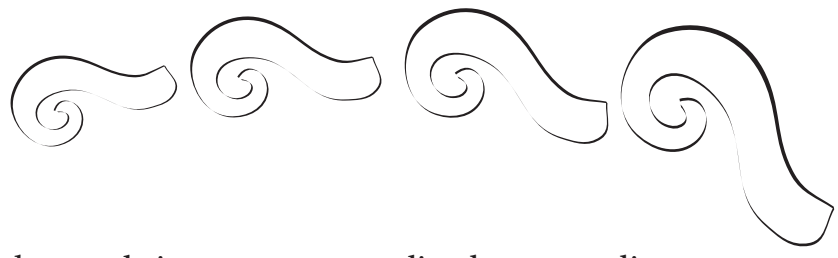

base al instrumento aplicado, nos dimos cuenta que los niños se logran identificar con los personajes de la telenovela ya mencionada, como lo son el personaje de la víctima o el personaje de la familia de la víctima. La mayoría de los alumnos mencionan que se identifican con estos roles porque han vivido historias similares a las presentadas en la telenovela.

El estereotipo de las mujeres de la telenovela La rosa de Guadalupe, según los alumnos es que consideran que las mujeres que aparecen en el programa referido se muestran cultas y educadas. Los alumnos aceptaron que a raíz de este programa han modificado su forma de vestir porque quieren estar a la moda como se muestra en la telenovela. Con estas respuestas comprobamos una vez más la teoría de Elihu Katz " La teoria de los usos y gratificaciones" (Estados Unidos, 1960) con su postulado de las $<<$ Necesidades intra-psicológicas $>>$ : que se refiere al identificarse, al autoconocimiento y a la identificación de valores que se presentan, así como relacionarse con algún estereotipo.

Por último el mensaje que le da la telenovela es que la vida está llena de peligros y dificultades. Y les genera desconfianza hacia la sociedad. (Katz, Elihu, Estados Unidos 1960) "Cuestiona la relación directa entre estímulo y respuesta, atendiendo al hecho de que cada uno de los destinatarios de un mismo mensaje, viene precedido por un contexto el cuál condiciona el efecto de dicho mensaje. Por tanto, no son solo los estímulos los que ponen en marcha el proceso comunicativo, sino los propios receptores al elegir el contenido e interpretarlo. Los estímulos generan unos efectos tan sólo si el individuo quiere responder a ellos."

\section{Analiza el cumplimiento de objetivos.}

Los objetivos de la investigación se cumplen en un $100 \%$, pues los resultados del instrumento contestaron nuestros objetivos específicos: 


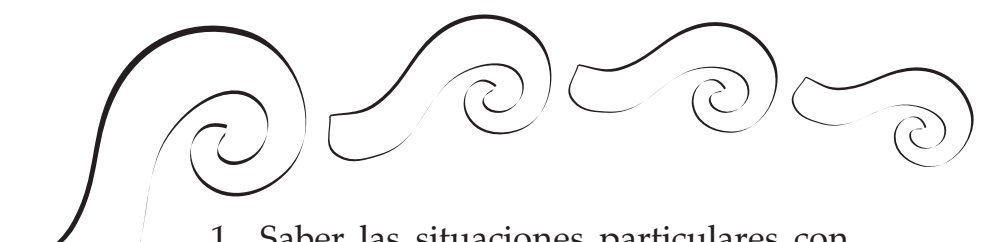

1. Saber las situaciones particulares con las que se identifican los alumnos de $2^{\mathrm{o}}$ de la telesecundaria de Tilcuautla con el programa de La rosa de Guadalupe.

Se cumple el objetivo ya que los alumnos mencionaron que se identificaban con las situaciones que vivían las víctimas de la telenovela o con la familia de la víctima.

2. Clasificar el contenido del programa de La Rosa de Guadalupe.

Los alumnos consideran que el contenido del programa es violencia, discriminación y bulliyng, considerados problemas actuales de la sociedad mexicana.

3. Identificar los estereotipos que más fuerza tienen sobre los alumnos de $2^{\mathrm{o}}$ de la telesecundaria de Tilcuautla.

Los estereotipos que se mencionan en las encuestas sobre las mujeres pues consideran que son educadas y cultas, así como amorosas y pobres. Otro estereotipo mencionado fue el del personaje rico o el que produce el daño, aunque para la mayoría no son importantes los personajes como para imitarlos.

\section{Aportaciones de la investigación.}

- La mayoría de los adolescentes de entre 13 y 15 de la Telesecundaria 196 de segundo grado de secundaria observan casualmente La rosa de Guadalupe. Esto nos ayuda a saber que la manipulación que puede ejercer este programa puede ser limitada debido a la falta de exposición hacia éste.

- Encontramos que los adolescentes se identifican con algunas circunstancias, ya sea con los familiares de la víctima, o la víctima. Esto significa que los programas de televisión, éste en específico, sé causa que la gente se identifique, así como que se compare y actúe conforme a lo que observa.

- Según la encuesta, los adolescentes creen que las mujeres que aparecen en La rosa de Guadalupe son cultas y educadas.

- La mayoría de programas van enfocados a la violencia. El programa da mucho contenido violento.

- El 50\% de los adolescentes adquiere un gusto personal por la moda, debido a la televisión.

- Los adolescentes creen que el mensaje que se queda en su mente es negativo: "la vida está llena de problemas". Y esto nos ayuda a entender que este programa les da una perspectiva pesimista hacia la vida.

\section{Limitaciones}

Nos encontramos con muy pocas limitaciones a la hora de aplicar el instrumento.

1. La seriedad presentada en las encuestas por parte de los alumnos fue poca.

Para evitar la indiferencia tratamos de explicarles el procedimiento para que los resultados fueran los óptimos, de manera que tratamos que todos los apartados de las encuestas fueran contestados.

2. Aplicamos un primer instrumento (cuestionario) y a la hora de hacer las conclusiones nos dimos cuenta que no cumplían nuestros objetivos, ni nuestras preguntas de investigación, después aplicamos un segundo cuestionario que si respondía a nuestras preguntas.

3. El día que íbamos a aplicar el segundo cuestionario, la escuela estaba cerrada y 
fuimos al día siguiente, pero el tiempo que teníamos ya era muy poco.

\section{Recomendaciones}

- Una de las recomendaciones sería enviar una carta a la producción del programa para hacerlos conscientes que la mayoría de sus programas dejan un mensaje negativo para los adolescentes.

- Explicarles a los adolescentes que ficticia e irreal puede ser la televisión.

- Acudir a los papás para que puedan ayudar a sus hijos en los problemas de sus hijos, concienciarlos para que ayuden a sus hijos.

- Localizar qué adolescentes han sido influenciados más negativamente, y recomendar visitas al psicólogo.

- Realizar actividades con los adolescentes que los mantengan ocupados en metas y proyectos que ayuden en su desenvolvimiento y a su autoestima, y que permitan que el adolescente vea sus logros.

- Recomendar programas de televisión más productivos.
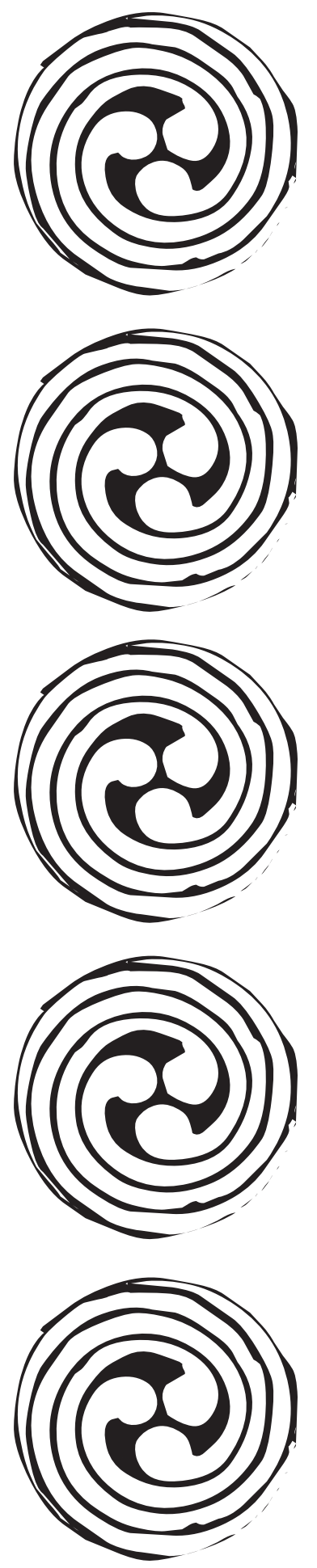


\section{Fuentes de consulta:}

M. Wolf (1991) La investigación de la comunicación de masas. Crítica y perspectivas, Barcelona, España: Paidós.

Gustavo Gili, "Estructura y función de la comunicación en sociedad", en M. de Moragas (ed). Sociología de la comunicación de masas, Barcelona 1986, Vol. II

De Flour, Melvin (1997). Teorías de la comunicación de masas. Ed Paidos.

Mc Quail, Denis. Introducción a la teoría de la comunicación de masas. Ed. Paidos.

Portillo Sánchez Maricela (1998), Espacios de comunicación. Universidad Iberoamericana, Cd de México, México.

Gloria Robles. http://www.proyectopv.org/1-verdad/manipulacion.htm

Jorge González Treviño. http://definicion.de/television/

Televisa. http://televisa.esmas.com/la-rosa-de-guadalupe/

RAE. http://lema.rae.es/drae/?val=prototipo

A y M, Maklelart (1997), Historia de las teorías de la comunicación, España Ed Paidós.

Inegi. http://www.inegi.org.mx/movil/
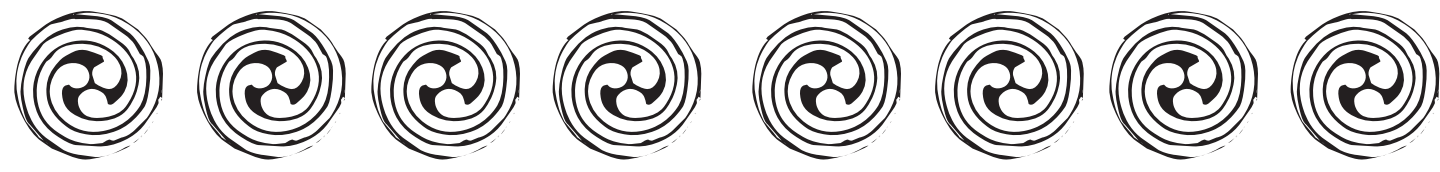

$$
\text { ๑107@ }
$$




\section{Anexos}

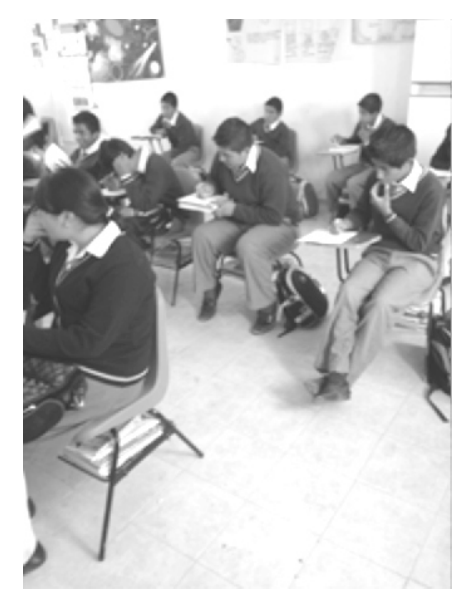

Miércoles 5/05/13 Alumnos contestando la entrevista.

FOTO: Maria Fernanda Jiménez González.

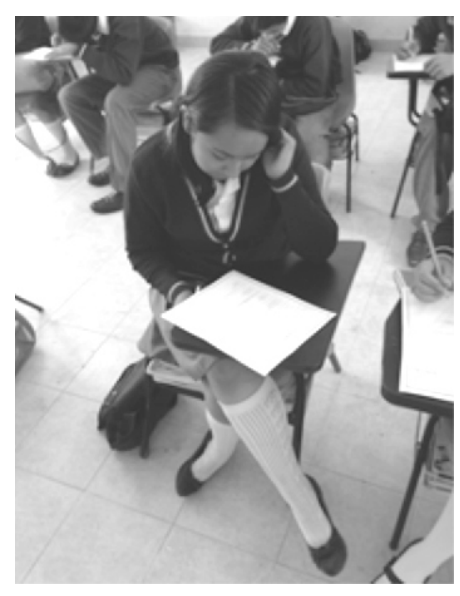

Miércoles 5/05/13 Alumnos contestando la entrevista.

FOTO: Maria Fernanda Jiménez González

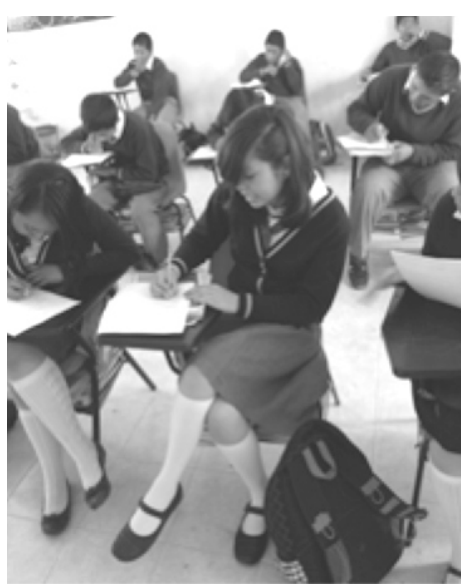

Miércoles 5/05/13 Alumnos contestando la entrevista.

FOTO: Maria Fernanda Jiménez González.

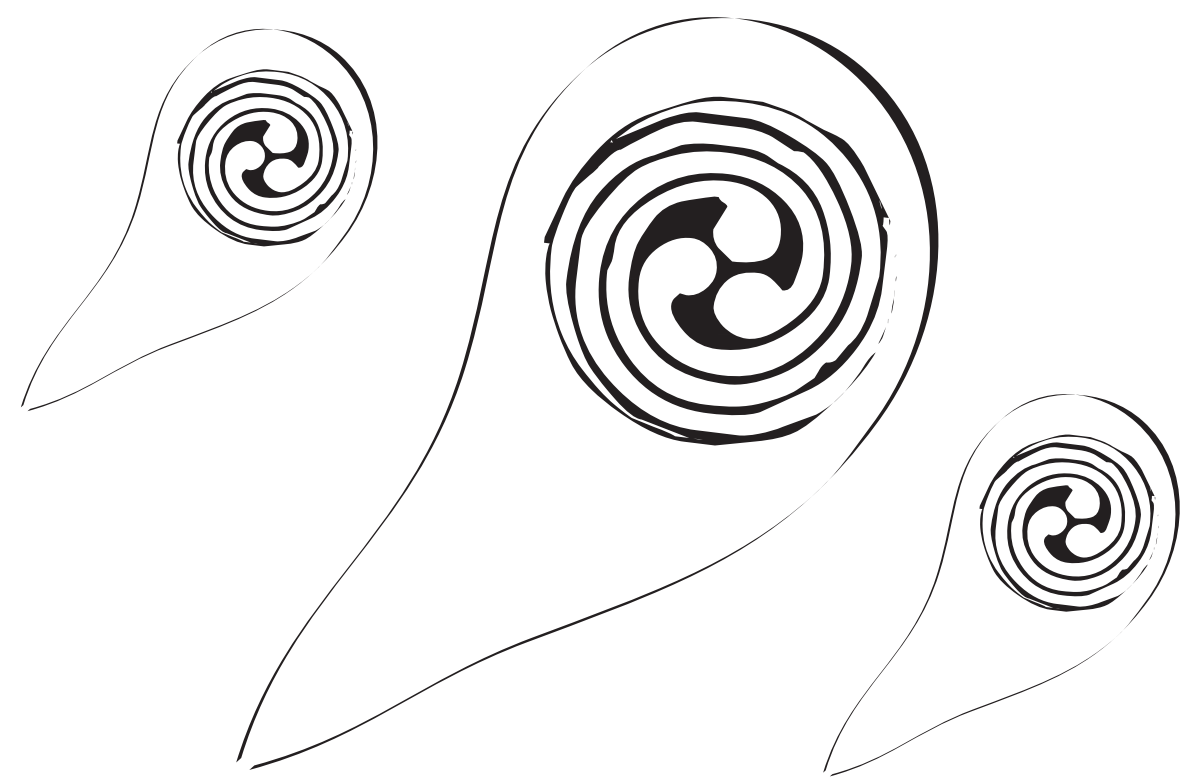

